\title{
Inverse association between soya food consumption and insulin resistance in Japanese adults
}

\author{
Mariko Nakamoto 1,2,3, Hirokazu Uemura ${ }^{1}$, Tohru Sakai², Sakurako Katsuura-Kamano ${ }^{1}$, \\ Miwa Yamaguchi ${ }^{1}$, Mineyoshi Hiyoshi ${ }^{1,}{ }^{1}$ and Kokichi Arisawa ${ }^{1, *}$ \\ 'Department of Preventive Medicine, Institute of Health Biosciences, The University of Tokushima Graduate School, \\ 3-18-15 Kuramoto-cho, Tokushima 770-8503, Japan: ${ }^{2}$ Department of Public Health and Applied Nutrition, Institute \\ of Health Biosciences, The University of Tokushima Graduate School, Tokushima, Japan: ${ }^{3}$ Research Fellow of the \\ Japan Society for the Promotion of Science
}

Submitted 18 February 2014: Final revision received 13 August 2014: Accepted 3 October 2014: First published online 10 November 2014

\begin{abstract}
Objective: The purpose of the present study was to examine the association between soya food consumption and insulin resistance using baseline data of the Japan Multi-Institutional Collaborative Cohort (J-MICC) Study in Tokushima, Japan. Design: This cross-sectional study included 1274 subjects, aged 34-70 years at baseline, living in Tokushima Prefecture between 2008 and 2013. Fasting blood samples were collected and information on lifestyle characteristics including soya food intake and medical history were obtained using a structured selfadministered questionnaire. The homeostasis model assessment of insulin resistance (HOMA-IR) was measured and those with HOMA-IR $\geq 2.5$ were defined as having insulin resistance. Multiple logistic regression models were used to analyse the association between soya product intake and the prevalence of insulin resistance.

Setting: Rural communities located in Tokushima Prefecture, Japan, between 2008 and 2013.

Subjects: A total of 1148 adults (565 men and 583 women), aged 34-70 years.

Results: The frequency of intake of miso soup, total non-fried soya products and total soya products showed significant inverse dose-response relationships with insulin resistance, after adjustments for potential confounders. When soya product intake was calculated as soya protein and isoflavone, the odds ratios of insulin resistance decreased significantly as the estimated intake of soya protein increased. Furthermore, significant inverse dose-response relationships were observed for total non-fried soya products and total soya products, after adjustment for total vegetable or total fibre consumption.

Conclusions: The present results indicate that the intake of soya products and nonfried soya products is associated with reduced insulin resistance in the Japanese population.
\end{abstract}

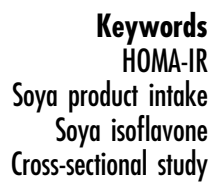

Type 2 diabetes is associated with severe complications including retinopathy, nephropathy, neuropathy, stroke and CVD, and has become a worldwide concern for public health. The number of patients with diabetes worldwide has been estimated to increase from 171 million in 2000 to 366 million by the year $2030^{(1)}$. An increased incidence rate of type 2 diabetes has also been reported in both adults and children during the past several decades ${ }^{(2,3)}$.

\footnotetext{
$\dagger$ Present address: Department of Biochemistry, Shimane University
} Faculty of Medicine, 89-1 Izumo, Shimane 693-8501, Japan.
Diabetes is characterized by a hyperglycaemic condition due to insulin hyposecretion or insulin resistance at target organs, including adipose tissue, liver and muscle. Insulin resistance has been aetiologically linked to a pro-inflammatory state ${ }^{(4)}$. Inflammatory pathways in insulin resistance can be initiated by extracellular mediators such as cytokines and NEFA, or by intracellular stresses such as the excessive production of reactive oxygen species ${ }^{(5,6)}$.

Soyabean is a member of the legume family and is a part of the traditional Asian diet. Soyabean and soya products are rich sources of various nutrients such as plant protein, fibre, vitamins, minerals and phyto-oestrogens (isoflavones) ${ }^{(7)}$. 
Isoflavones may exert beneficial effects on glucose homeostasis because they structurally resemble oestradiol. Previous experimental studies have reported that soyabean phytochemical extracts inhibit glucose uptake in the intestine ${ }^{(8)}$ and isoflavones have been shown to directly stimulate insulin production in pancreatic islet $\beta$ cells through the cyclic adenosine monophosphate pathway ${ }^{(9)}$. Furthermore, the administration of soya protein and isoflavones led to increased insulin production, improved glucose metabolism $^{(10)}$ and lower insulin requirements ${ }^{(11)}$ in animals.

An inverse association between soya food intake and type 2 diabetes has been reported in several, but not all human studies. A diet with soyanuts ${ }^{(12)}$ and supplements with soya protein, fibre, isoflavone and other soya products improved glucose homeostasis in patients with type 2 diabetes ${ }^{(13,14)}$. An inverse association between soya food intake and the prevalence of glycosuria was also reported in postmenopausal non-obese Chinese women ${ }^{(15)}$. Furthermore, a recent prospective study in Japan showed that higher intake of soya products and isoflavones was associated with a decreased odds ratio of diabetes among overweight or postmenopausal women ${ }^{(16)}$. The consumption of some soya foods was inversely associated with the risk of type 2 diabetes among Asians in two prospective studies $^{(17,18)}$. On the other hand, soya intake was positively associated with the risk of diabetes in another cohort study in Hawaii ${ }^{(19)}$.

To date, no studies have examined the association between soya intake and insulin resistance in Japan. Therefore, we investigated the association between soya product intake and insulin resistance levels in the Japanese population.

\section{Methods}

\section{Study population}

The present study population included participants in the Japan Multi-Institutional Collaborative Cohort (J-MICC) Study, which intends to examine associations between lifestyle and genetic factors and their interactions with lifestyle-related diseases. Details of the survey method have been reported elsewhere ${ }^{(20)}$. The study population consisted of two groups. The first group (group 1) comprised 577 men and women who had attended the Tokushima Prefectural General Health Check-up Center between 23 January 2008 and 24 November 2011 and agreed to participate in the J-MICC Study (response rate $=14.8 \%$ ). The second group (group 2) consisted of 697 men and women living in Tokushima city. We distributed approximately 98700 leaflets explaining the objective and the method of the J-MICC Study all over Tokushima city (total population $=264500$ ) from July 2012 to February 2013. These 697 individuals read the leaflet and attended the health check-ups performed by our research team between 25 July 2012 and 27 February 2013. From the total of 1274 men and women (groups 1+2) aged 34-70 years, we excluded individuals with a previous history of stroke ( $n$ 14), IHD ( $n$ 29), medical history of or medical treatment for diabetes ( $n$ 68), those whose data on the health checkup ( $n$ 10) or soya products ( $n$ 2) were not available and those whose daily total energy intake was low $(<4189 \mathrm{~kJ} / \mathrm{d})$ or high $(>16756 \mathrm{~kJ} / \mathrm{d} ; n 10)^{(21)}$. The remaining 1148 individuals ( 565 men and 583 women) were used for analysis in the present study (Fig. 1). Participation was essentially voluntary and after explaining the details of the study, we obtained written informed consent from each participant. The study protocol was reviewed and approved by the Ethics Committees of the authors' institutions.

\section{Questionnaires and calculation of the intake of soya protein and soya isoflavone}

Study participants were requested to complete a structured self-administered questionnaire, including questions on physical activity during leisure time, frequency of intake of foods and beverages, smoking and drinking habits, and current and previous history of diseases.

Participants answered a validated, short FFQ on their dietary habits, which asked how often they had consumed forty-seven items of food/recipes and beverages over the past year ${ }^{(22-25)}$. The questionnaire included four items on soya product consumption: miso soup $(=1)$; tofu $(=2)$; fermented soyabeans and soyabeans $(=3)$; and fried tofu mixed with vegetables, fried bean curd and thick deepfried tofu (hereafter 'deep-fried tofu'; = 4). The frequency of soya product intake was classified into the following eight categories: ' 3 times/day' (21/week), 'twice/day' (14/week), 'once/day' (7/week), '5-6 times/week' (5-6/week), '3-4 times/week' (3·5/week), '1-2 times/week' (1.4/week), '1-3 times/month' (0.7/week) and 'almost never' (0/week). The total frequency of non-fried soya product intake was calculated as the sum of miso soup, tofu and boiled or fermented soyabeans $(1+2+3)$. The total frequency of soya product intake was calculated as the sum of miso soup, tofu, boiled or fermented soyabeans and deep-fried tofu $(1+2+3+4)$.

Since the portion size of each soya product was not queried, the intake per meal from four $3 \mathrm{~d}$ diet records, which were collected within an interval of 4 months or shorter in a group of twenty-eight participants, was used to calculate the weekly intake of soya products. The portion sizes of miso soup (1), tofu (2), boiled or fermented soyabeans (3) and deep-fried tofu (4) were 9.9 (the amount used as miso), 53.8, 31.7 and $19.5 \mathrm{~g} / \mathrm{meal}$, respectively. Total soya protein intake was estimated by summing the soya protein contained in each specific soya food on the basis of the Standard Tables of Food Composition in Japan (Ministry of Education, Culture, Sports, Science and Technology, 2010). Total soya isoflavone intake was estimated by summing the soya isoflavone contained in each soya food according to estimates by the Ministry of Agriculture, Forestry and Fisheries of Japan. 


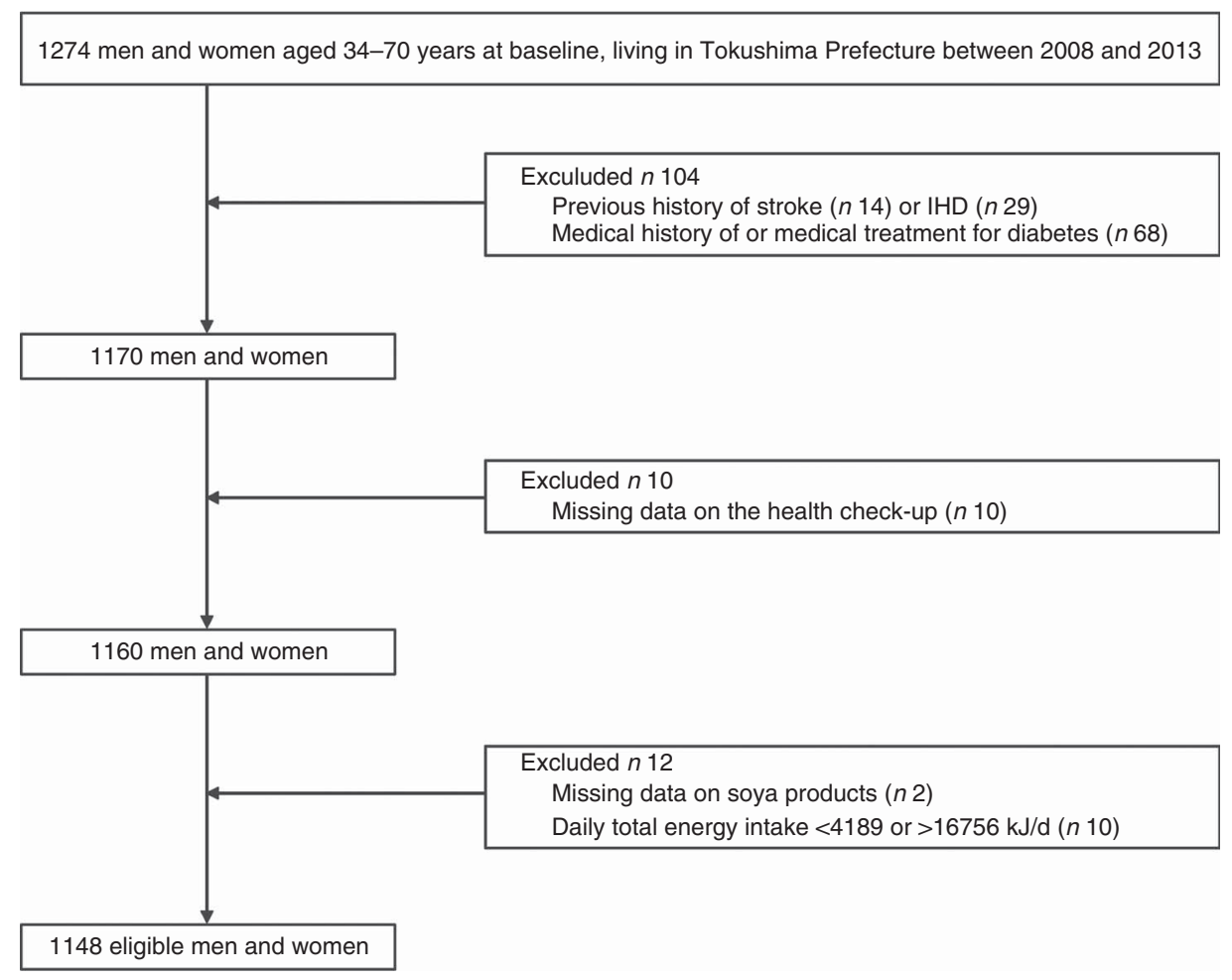

Fig. 1 An overview of the analysed individuals. Among 1274 men and women aged 34-70 years at baseline, living in Tokushima Prefecture between 2008 and 2013, who participated, 1148 eligible individuals (565 men and 583 women) were finally analysed

The total energy intake of each participant was estimated using a program developed at the Department of Public Health, Nagoya City University School of Medicine ${ }^{(22,23)}$. Physical activity during leisure time was evaluated by multiplying the frequency and level of exercise-related metabolism, which consisted of three steps, summed and expressed as MET-h/week (where MET is metabolic equivalent of task). The three steps of exercise-related metabolism level were light (including walking and golf, 3.4 MET), moderate (including jogging and swimming, 7.0 MET) and heavy exercise (including marathon running and fighting sports, 10.0 MET).

\section{Anthropometric and biochemical measurements}

Body height, weight, waist circumference, blood pressure, fasting plasma glucose and serum levels of TAG and HDL cholesterol were obtained at the time of health check-ups. Participants were requested not to eat after 20.00 hours, and received a medical check-up from 08.00 to 11.30 hours the following day. Serum insulin was measured using a chemiluminescence immunoassay at BML Inc. (Tokyo, Japan). The homeostasis model assessment of insulin resistance (HOMA-IR) was calculated using the following equation: fasting insulin $(\mathrm{mU} / \mathrm{l}) \times$ fasting plasma glucose $(\mathrm{g} / \mathrm{dl}) / 405$. Insulin resistance was defined as HOMA-IR $\geq 2 \cdot 5$.

\section{Statistical analysis}

Continuous variables were expressed as mean and standard deviation, whereas those with skewed distribution were expressed as median and 25 th-75th percentile. The one-way ANOVA, Kruskal-Wallis test or $\chi^{2}$ test was used to determine significant differences between the characteristics of participants according to total soya products consumption. The associations of soya food consumption with the prevalence of insulin resistance were examined using multiple logistic regression analysis, after adjustment for gender, age (continuous), recruitment (binary), family history of type 2 diabetes mellitus (without a family history, unclear and with a family history) in father and mother, total energy intake (quartiles), physical activity (quartiles), smoking (current, no and ex-smokers) and drinking habits (current drinkers and others) in model 1, and additionally for BMI (continuous) in model 2. All multivariate models were further adjusted for total vegetable intake (quartiles) or total fibre intake (quartiles). Furthermore, in multiple logistic regression analysis, we adjusted for menopause status (men, premenopausal women, perimenopausal women and postmenopausal woman) in place of gender. The consumption of miso soup, tofu, total non-fried soya products and total soya products was divided into quartiles, with the first quartile being defined as the reference. Dummy variables were created for categorical variables and those except for reference categories were included in the models. Odds ratios and $95 \%$ profile likelihood confidence intervals were calculated. We conducted tests for trend using the median value of each quartile for the consumption of each soya food and the likelihood ratio test in logistic models. 
All statistical tests were based on two-sided probabilities and performed using the SAS statistical software package version 8.2. All $P$ values $<0.05$ were considered significant ${ }^{(26)}$.

\section{Results}

\section{Characteristics of the participants}

The total number of participants was 1148 (565 men and 583 women). The mean age was 52.6 (SD 9.9) years in men and 52.0 (SD 9.8) years in women. Table 1 shows the characteristics of the participants according to the frequency of total soya product intake. Age, physical activity, serum levels of HDL cholesterol and total energy intake increased as the consumption of total soya products $(1+2+3+4)$ increased. On the other hand, fasting insulin, HOMA-IR and the proportion of current smokers decreased with increasing total soya product intake. Table 1 also shows the estimated intake of soya protein and isoflavone, presented for two types of soya products $(1+2+3$ and $1+2+3+4)$, according to frequency of total soya product intake.

\section{Associations of the intake of each soya product with insulin resistance}

Gender-, age- and multivariate-adjusted associations between the consumption of each soya product and insulin resistance are presented in Table 2. After adjustments for age, gender, recruitment, family history of type 2 diabetes mellitus, total energy intake, physical activity, and smoking and drinking habits (model 1), the OR of insulin resistance decreased significantly as the consumption of miso soup, total non-fried soya products $(1+2+3)$ and total soya products $(1+2+3+4)$ increased ( $P$ for trend $<0 \cdot 05)$. On the other hand, the association between the intake of tofu and insulin resistance was rather U-shaped, although the $P$ for trend was $<0 \cdot 05$. To assess whether the association between soya product intake and insulin resistance was confounded or mediated by obesity, an analysis was performed with an additional adjustment for BMI (model 2). The relationships of miso soup, total nonfried soya products $(1+2+3)$ and total soya products $(1+2+3+4)$ with insulin resistance remained significant (Table 2, Fig. 2(a)).

\section{Associations of the estimated intake of soya protein and soya isoflavone with insulin resistance}

Table 3 shows the gender-, age-, and multivariate-adjusted associations between the estimated intake of soya protein and soya isoflavone from non-fried soya products $(1+2+3)$ and total soya products $(1+2+3+4)$ with insulin resistance. After adjustments for age, gender, recruitment, family history of type 2 diabetes mellitus, total energy intake, physical activity, and smoking and drinking habits (model 1), the OR of insulin resistance decreased significantly as the estimated intake of soya protein from non-fried soya products
$(1+2+3)$ and total soya products $(1+2+3+4)$, and isoflavone from total soya products $(1+2+3+4)$, increased $(P$ for trend $<0.05)$. On the other hand, the OR was lowest in the third quartile and the association between the intake of isoflavone contained in non-fried soya products $(1+2+3)$ and insulin resistance was U-shaped, although the $P$ for trend was $<0.05$. After an additional adjustment for BMI (model 2), the associations between the intake of soya protein from non-fried soya products $(1+2+3)$ and total soya products $(1+2+3+4)$ with insulin resistance were significant (Fig. 2(b)).

To assess whether the associations between the intake of soya products and insulin resistance were confounded by other factors, menopausal status, total vegetable intake or total fibre intake was further adjusted (data not shown). After adjustment for menopausal status, the results were essentially similar. When total vegetable intake was adjusted, the inverse association remained significant for miso soup, total non-fried soya products and total soya products. On the other hand, after adjustment for total fibre intake, the associations were significant for total non-fried soya product and total soya product intake, regardless of adjustment for BMI.

\section{Discussion}

In the present study we showed that a higher frequency of consumption of total non-fried soya products and total soya products was associated with reduced OR of insulin resistance among a Japanese population. When soya product intake was calculated as soya protein and soya isoflavone, the associations were slightly attenuated.

The association between the high intake of soya products and decreased OR of insulin resistance observed in the present study was not unexpected. The consumption of soya foods (tofu and other soya products) was previously associated with a significantly reduced prevalence of glycosuria in 39385 Chinese women aged 40-70 years without diabetes, especially postmenopausal women with BMI $<25$ $\mathrm{kg} / \mathrm{m}^{2(15)}$. Nanri et al. reported a significant correlation between soya products and daidzein intake and a reduced cumulative incidence of type 2 diabetes among women with $\mathrm{BMI}>25 \mathrm{~kg} / \mathrm{m}^{2}$ and postmenopausal women ${ }^{(16)}$. In a prospective study in China, Villegas et al. reported that the consumption of legumes and soya foods was associated with a decreased risk of type 2 diabetes in 64227 women aged 40-70 years ${ }^{(17)}$. Mueller et al. also observed that while unsweetened soya product intake was protective against diabetes, no significant correlation between the intake of soya-derived components and insulin resistance was observed in 43176 Chinese Singaporeans ${ }^{(18)}$. On the other hand, higher soya food intake was associated with a slightly elevated diabetes risk in three ethnic groups in a recent cohort study performed in the USA, and this increased risk was limited to overweight and obese subjects ${ }^{(19)}$. Some 
Table 1 Characteristics of the participants according to intake frequency of total soya products; 565 men and 583 women aged $34-70$ years baseline, living in Tokushima Prefecture between 2008 and 2013, Japan Multi-Institutional Collaborative Cohort (J-MICC) Study

\begin{tabular}{|c|c|c|c|c|c|c|c|c|c|}
\hline & \multicolumn{2}{|c|}{ Q1 (n 312) } & \multicolumn{2}{|c|}{ Q2 ( $n$ 282) } & \multicolumn{2}{|c|}{ Q3 (n 280) } & \multicolumn{2}{|c|}{ Q4 (n 274) } & \multirow[b]{2}{*}{$P$} \\
\hline & $\begin{array}{c}n, \text { mean } \\
\text { or median }\end{array}$ & $\begin{array}{l}\% \text {, sD or } \\
\text { P25-P75 }\end{array}$ & $\begin{array}{l}n, \text { mean } \\
\text { or median }\end{array}$ & $\begin{array}{l}\% \text {, sD or } \\
\text { P25-P75 }\end{array}$ & $\begin{array}{c}n, \text { mean } \\
\text { or median }\end{array}$ & $\begin{array}{c}\%, \text { sD } \\
\text { or P25-P75 }\end{array}$ & $\begin{array}{l}n, \text { mean } \\
\text { or median }\end{array}$ & $\begin{array}{c}\% \text {, sD } \\
\text { or P25-P75 }\end{array}$ & \\
\hline \multicolumn{10}{|l|}{$\operatorname{Sex}^{\star}$} \\
\hline Men & 152 & 48.7 & 148 & 52.5 & 146 & 52.1 & 119 & 43.4 & 0.12 \\
\hline Women & 160 & $51 \cdot 3$ & 134 & 47.5 & 134 & 47.9 & 155 & 56.6 & \\
\hline Age (years) $\dagger$ & 50.5 & 9.6 & 50.6 & 9.9 & 52.9 & 9.4 & 55.7 & 9.6 & $<0.001$ \\
\hline BMI $\left(\mathrm{kg} / \mathrm{m}^{2}\right) \dagger$ & 23.5 & 3.6 & 23.1 & 3.3 & 23.1 & 3.4 & 23.0 & 3.3 & 0.22 \\
\hline Physical activity (MET-h/week)‡ & 3.8 & $0.40-17.9$ & 4.0 & $0.40-16.4$ & 7.7 & $1 \cdot 2-18.4$ & 12.6 & $3.0-30.6$ & $<0.001$ \\
\hline Waist circumference $(\mathrm{cm}) \dagger$ & 82.8 & 10.4 & 82.0 & 9.6 & $82 \cdot 1$ & $10 \cdot 6$ & 81.4 & 9.2 & 0.42 \\
\hline Systolic blood pressure $(\mathrm{mmHg}) \dagger$ & $124 \cdot 1$ & 18.7 & 122.8 & 18.6 & 123.4 & 18.4 & 124.7 & 17.8 & 0.63 \\
\hline Diastolic blood pressure $(\mathrm{mmHg}) \dagger$ & 75.6 & 12.6 & 74.8 & 12.5 & 74.2 & $12 \cdot 2$ & 75.0 & 11.2 & 0.57 \\
\hline TAG $(\mathrm{mg} / \mathrm{dl}) \neq$ & 91 & $64 \cdot 5-130$ & 87 & $60 \cdot 0-123$ & 87 & $63 \cdot 0-122$ & 80.5 & $57 \cdot 0-126$ & 0.30 \\
\hline $\mathrm{HDL}$ cholesterol $(\mathrm{mg} / \mathrm{dl}) \dagger$ & 58.4 & 15.7 & $60 \cdot 2$ & 14.9 & 58.8 & 14.7 & 62.8 & 17.4 & 0.003 \\
\hline Fasting plasma glucose $(\mathrm{mg} / \mathrm{dl}) \dagger$ & 92.9 & $12 \cdot 7$ & 94.5 & $15 \cdot 1$ & 94.6 & 10.7 & 93.4 & 9.7 & 0.25 \\
\hline Fasting insulin $(\mathrm{mU} / \mathrm{l}) \ddagger$ & $5 \cdot 2$ & $3.8-7.9$ & 5.0 & $3 \cdot 5-7 \cdot 2$ & 5.0 & $3.5-7.6$ & $4 \cdot 3$ & $3.1-5.9$ & $<0.001$ \\
\hline HOMA-IR $\ddagger$ & 1.2 & $0.84-1.8$ & 1.1 & $0.76-1.8$ & 1.2 & $0.78-1.9$ & 0.96 & $0.69-1 \cdot 4$ & $<0.001$ \\
\hline \multicolumn{10}{|l|}{ Place of recruitment ${ }^{*}$} \\
\hline Tokushima Prefectural General Health Check-up Center & 122 & 39.1 & 133 & 47.2 & 139 & 49.6 & 123 & 44.9 & 0.06 \\
\hline Western area in Tokushima Prefecture & 190 & 60.9 & 149 & 52.8 & 141 & 50.4 & 151 & $55 \cdot 1$ & \\
\hline \multicolumn{10}{|l|}{ Father's history of type 2 diabetes mellitus* } \\
\hline Without a family history & 210 & 67.3 & 186 & 66.0 & 197 & $70 \cdot 4$ & 202 & 73.7 & 0.11 \\
\hline Unclear & 60 & 19.2 & 52 & 18.4 & 35 & 12.5 & 41 & 15.0 & 0.11 \\
\hline \multirow{2}{*}{\multicolumn{10}{|c|}{$\begin{array}{l}\text { With a family history } \\
\text { Mother's history of type } 2 \text { diabetes mellitus* }\end{array}$}} \\
\hline & & & & & & & & & \\
\hline Without a family history & 239 & 76.6 & 212 & 75.2 & 223 & 79.6 & 209 & $76 \cdot 3$ & 0.84 \\
\hline Unclear & 41 & 13.1 & 38 & 13.5 & 27 & 9.6 & 34 & 12.4 & 007 \\
\hline With a family history & 32 & 10.3 & 32 & 11.4 & 30 & 10.7 & 31 & 11.3 & \\
\hline \multicolumn{10}{|l|}{ Smoking habit ${ }^{\star}$} \\
\hline Current & 70 & 22.4 & 49 & 17.4 & 44 & 15.7 & 27 & 9.9 & 0.002 \\
\hline Past & 67 & 21.5 & 76 & 27.0 & 81 & 28.9 & 67 & 24.5 & \\
\hline Never & 175 & 56.1 & 157 & 55.7 & 155 & 55.4 & 180 & 65.7 & \\
\hline \multicolumn{10}{|l|}{ Drinking habit* } \\
\hline Current & 160 & $51 \cdot 3$ & 162 & 57.4 & 167 & 59.6 & 139 & 50.7 & 0.16 \\
\hline Past & 8 & 2.6 & 5 & 1.8 & 2 & 0.7 & 4 & 1.5 & 0.70 \\
\hline Never & 144 & $46 \cdot 2$ & 115 & 40.8 & 111 & 39.6 & 131 & 47.8 & \\
\hline Total energy intake $(\mathrm{kJ} / \mathrm{d}) \dagger$ & 6833 & 1497 & 6998 & 1317 & 7359 & 1497 & 7304 & 1443 & $<0.001$ \\
\hline \multicolumn{10}{|l|}{ Frequency of each soya product intake (times/week) } \\
\hline (1) Miso soup (times/week)‡ & 1.4 & $0.7-1.4$ & 3.5 & $1.4-3.5$ & 5.6 & $3.5-7.0$ & 7.0 & $5.6-7.0$ & $<0.001$ \\
\hline (2) Tofu (times/week) $\ddagger$ & 0.7 & $0.7-1 \cdot 4$ & 1.4 & $0.7-1 \cdot 4$ & 1.4 & $1.4-3.5$ & 3.5 & $1.4-5 \cdot 6$ & $<0.001$ \\
\hline (3) Boiled or fermented soyabeans (times/week)‡ & 0.7 & $0.35-1.1$ & 0.7 & $0.7-1 \cdot 4$ & 1.4 & $0.7-1.4$ & 3.5 & $1 \cdot 4-7 \cdot 0$ & $<0.001$ \\
\hline $\begin{array}{l}\text { (4) Deep-fried tofu (times/week) } \ddagger \\
\text { (1) D }\end{array}$ & 0.7 & $0.0-0.7$ & 0.7 & $0.7-1.4$ & 0.7 & $0.7-1.4$ & 1.4 & $0.7-3.5$ & $<0.001$ \\
\hline$(1+2+3)$ Total non-fried soya products (times/week) $\ddagger, \S$ & 2.8 & $2 \cdot 1-3 \cdot 5$ & 5.6 & $4 \cdot 9-6 \cdot 3$ & 8.4 & $7 \cdot 7-9 \cdot 1$ & 14.0 & $11 \cdot 9-16.8$ & $<0.001$ \\
\hline$(1+2+3+4)$ Total soya products (times/week)‡,\|l & 3.5 & $2 \cdot 8-4 \cdot 2$ & 7.0 & $6 \cdot 3-7 \cdot 0$ & 9.8 & $9.1-10.5$ & $15 \cdot 4$ & 13.3-18.2 & $<0.001$ \\
\hline \multicolumn{10}{|l|}{ Soya protein consumption (g/week) } \\
\hline$(1+2+3)$ Total non-fried soya products calculated on soya protein $(\mathrm{g} /$ week $) \ddagger, \S, \uparrow$ & $7 \cdot 3$ & $6 \cdot 1-9 \cdot 7$ & $13 \cdot 0$ & 10.4-15.4 & 18.6 & $15 \cdot 6-23 \cdot 5$ & $36 \cdot 3$ & $27 \cdot 6-46 \cdot 8$ & $<0.001$ \\
\hline$(1+2+3+4)$ Total soya products calculated on soya protein $(g /$ week $) \neq, \|$, , & $9 \cdot 3$ & $7 \cdot 3-12 \cdot 8$ & 15.4 & $12 \cdot 2-18 \cdot 2$ & 21.8 & $18 \cdot 8-26 \cdot 1$ & 40.1 & $32 \cdot 2-51 \cdot 1$ & $<0.001$ \\
\hline \multicolumn{10}{|l|}{ Soya isoflavone consumption (mg/week) } \\
\hline$(1+2+3)$ Total non-fried soya products calculated on soya isoflavone $(\mathrm{mg} /$ week) $), \S,{ }^{, *}$ & 29.6 & $23 \cdot 1-39 \cdot 9$ & $52 \cdot 2$ & $40 \cdot 6-62 \cdot 6$ & 73.3 & $63 \cdot 6-84 \cdot 9$ & $140 \cdot 9$ & $108 \cdot 6-201 \cdot 0$ & $<0.001$ \\
\hline 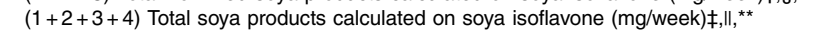 & 34.9 & $26 \cdot 1-46 \cdot 8$ & 58.9 & $46.4-67.9$ & 83.1 & $71 \cdot 2-92 \cdot 7$ & $154 \cdot 2$ & $119 \cdot 2-211 \cdot 7$ & $<0.001$ \\
\hline
\end{tabular}

$\mathrm{Q}$, quartile; MET, metabolic equivalent of task; HOMA-IR, homeostasis model assessment of insulin resistance.

Values are presented as $n$ and \%

.

(P25-P75).

Doiled or fermented soyabeans.

Total soya product intake was soup, tofu, boiled or fermented soyabeans and deep-fried tofu.

"*Total soya product intake was estimated by summing the soya isoflavone of each specific soya food according to estimates of the Ministry of Agriculture, Forestry and Fisheries of Japan. 
Table 2 Association between the intake frequency of each soya product and insulin resistance* among 565 men and 583 women aged 34-70 years baseline, living in Tokushima Prefecture between 2008 and 2013, Japan Multi-Institutional Collaborative Cohort (J-MICC) Study

\begin{tabular}{|c|c|c|c|c|c|c|c|c|}
\hline & \multicolumn{7}{|c|}{ Intake frequency (times/week) } & \multirow[b]{3}{*}{$P$ for trenc } \\
\hline & \multirow{2}{*}{$\frac{\text { Q1 }}{\text { OR }}$} & \multicolumn{2}{|r|}{ Q2 } & \multicolumn{2}{|c|}{ Q3 } & \multicolumn{2}{|c|}{ Q4 } & \\
\hline & & OR & $95 \% \mathrm{Cl}$ & OR & $95 \% \mathrm{Cl}$ & OR & $95 \% \mathrm{Cl}$ & \\
\hline \multicolumn{9}{|l|}{ Miso soup (times/week)† } \\
\hline No. of cases/participants & $59 / 394$ & \multicolumn{2}{|c|}{$25 / 292$} & \multicolumn{2}{|c|}{$41 / 414$} & \multicolumn{2}{|c|}{$5 / 48$} & \\
\hline Age- and gender-adjusted model & 1.00 & 0.45 & $0.27,0.74$ & 0.50 & $0.32,0.77$ & 0.54 & $0.18,1.34$ & 0.014 \\
\hline Model $1 \S$ & 1.00 & 0.42 & $0.25,0.71$ & 0.44 & $0.27,0.69$ & 0.47 & $0.15,1.20$ & 0.004 \\
\hline Model 2\| & 1.00 & 0.50 & $0.27,0.90$ & 0.46 & $0.27,0.79$ & 0.31 & $0.09,0.91$ & 0.004 \\
\hline \multicolumn{9}{|l|}{ Tofu (times/week)ף } \\
\hline No. of cases/participants & $58 / 420$ & \multicolumn{2}{|c|}{$50 / 421$} & \multicolumn{2}{|c|}{$14 / 220$} & \multicolumn{2}{|c|}{$8 / 87$} & \\
\hline Age- and gender-adjusted modelł & 1.00 & 0.83 & $0.55,1.26$ & 0.46 & $0.24,0.83$ & 0.63 & $0.26,1.33$ & 0.041 \\
\hline Model $1 \S$ & 1.00 & 0.91 & $0.59,1.40$ & 0.47 & $0.24,0.86$ & 0.64 & $0.26,1.39$ & 0.046 \\
\hline Model 2\| & 1.00 & 0.88 & $0.54,1.45$ & 0.52 & $0.24,1.04$ & 0.56 & $0.21,1.36$ & 0.071 \\
\hline \multicolumn{9}{|c|}{ Total non-fried soya products (times/week) ${ }^{* *}$} \\
\hline No. of cases/participants & $51 / 321$ & \multicolumn{2}{|c|}{$31 / 279$} & \multicolumn{2}{|c|}{$28 / 273$} & \multicolumn{2}{|c|}{$20 / 275$} & \\
\hline Age- and gender-adjusted modelł & 1.00 & 0.57 & $0.35,0.93$ & 0.54 & $0.32,0.89$ & 0.38 & $0.22,0.66$ & $<0.001$ \\
\hline Model $1 \S$ & 1.00 & 0.54 & $0.32,0.89$ & 0.47 & $0.27,0.79$ & 0.35 & $0.19,0.63$ & $<0.001$ \\
\hline Model 2\| & 1.00 & 0.71 & $0.39,1.26$ & 0.53 & $0.28,0.99$ & 0.37 & $0.19,0.72$ & 0.002 \\
\hline \multicolumn{9}{|l|}{ Total soya products (times/week)†† } \\
\hline No. of cases/participants & $48 / 312$ & \multicolumn{2}{|c|}{$34 / 282$} & \multicolumn{2}{|c|}{$28 / 280$} & \multicolumn{2}{|c|}{$20 / 274$} & \\
\hline Age- and gender-adjusted modelł & 1.00 & 0.68 & $0.42,1 \cdot 10$ & 0.55 & $0.33,0.91$ & 0.43 & $0.24,0.75$ & $<0.001$ \\
\hline Model $1 \S$ & 1.00 & 0.63 & $0.38,1.04$ & 0.49 & $0.29,0.83$ & 0.38 & $0.21,0.68$ & $<0.001$ \\
\hline Model 2\| & 1.00 & 0.81 & $0.45,1.43$ & 0.55 & $0.29,1.02$ & 0.37 & $0.19,0.73$ & 0.002 \\
\hline
\end{tabular}

Q, quartile.

${ }^{*}$ Adjusted odds ratios and $95 \%$ profile likelihood confidence intervals.

†Miso soup (times/week): Q1, 0-1.4; Q2, 1.4-3.5; Q3, 3.5-7.0; Q4, > 7.0.

$\neq$ Age- and gender-adjusted model: adjusted for age (continuous), gender and recruitment (binary).

$\S$ Model 1: adjusted for age (continuous), gender, recruitment (binary), family history of type 2 diabetes mellitus (categorical), total energy intake (categorical), physical activity (categorical), smoking (categorical) and drinking habits (binary).

IIModel 2: adjusted for age (continuous), gender, recruitment (binary), family history of type 2 diabetes mellitus (categorical), total energy intake (categorical), physical activity (categorical), smoking (categorical), drinking habits (binary) and BMI (continuous).

ITTu (times/week): Q1, 0-0.70; Q2, 0.70-1.4; Q3, 1.4-3.5; Q4, > 3.5.

${ }^{\star \star}$ Total unsweetened and non-fried soya products (times/week): Q1, 0-4.2; Q2, 4.2-7.0; Q3, 7.0-9.8; Q4, > 9.8.

††Total soya products (times/week): Q1, 0-4.9; Q2, 4.9-7.7; Q3, 7.7-11.2; Q4, > 11.2.

randomized controlled trials have reported that soya products $^{(12)}$, soya protein ${ }^{(12-14)}$ and soya isoflavone ${ }^{(14)}$ improve glycaemic control and insulin sensitivity. However, others showed no significant effect of soya protein ${ }^{(27-29)}$ and soya isoflavone $e^{(27,30,31)}$.

The results of an experimental study suggested that soyabean protein has the potential to improve insulin resistance or ameliorate obesity by inhibiting lipogenesis and promoting lipolysis in the liver and adipose cells ${ }^{(32)}$. Furthermore, isoflavone is similar to endogenous oestrogen in structure and exhibits a weak oestrogen-like action by combining with the oestrogen receptor in various organs ${ }^{(32)}$. By adjusting the function of adipose cells and inhibiting lipoprotein lipase, oestrogen has been shown to adjust the number of adipose cells, adipose deposition and lipid production ${ }^{(33-35)}$.

Therefore, we assessed the association between the intake of soya protein and isoflavone and insulin resistance. However, the frequency of the consumption of total soya products showed a clearer linear association with insulin resistance than the estimated intake of soya protein or isoflavones. One reason for this result may be that an in vivo metabolism of isoflavones was not considered in the present study. The soya isoflavones, such as daidzein and genistein, are phyto-oestrogens metabolized extensively by the intestinal microflora ${ }^{(36)}$. Some previous reports have suggested that $30-50 \%$ of Japanese have isoflavoneproducing ability depending on the intestinal bacteria ${ }^{(37)}$ and this ability is increased by dietary habits such as higher consumption of fibre, green tea and fish oil ${ }^{(38)}$. Therefore, there may be a discrepancy between the estimated intake of soya isoflavone and the actually metabolized amount of soya isoflavone. In addition, according to a recent metaanalysis, the purified or isolated components of soya (isoflavones or soya protein) were not as effective as whole soya foods in improving glycaemic control ${ }^{(39,40)}$. The reasons for this may include the presence of other components of soya, such as fibre, saponin, polysaccharides, phytosterol and unsaturated fatty acids, or their interactions. Thus, various experimental and human studies have reported the beneficial effects of soya or nutrient components of soya on glucose metabolism. However, the effects and mechanisms of soya and soya foods on insulin resistance have not been fully clarified and warrant further study.

After adjustment for BMI, the OR and $P$ for trend for the association between the intake of soya products, soya protein or soya isoflavone were not greatly altered (model $1 v$. model 2 in Tables 2 and 3). These results 


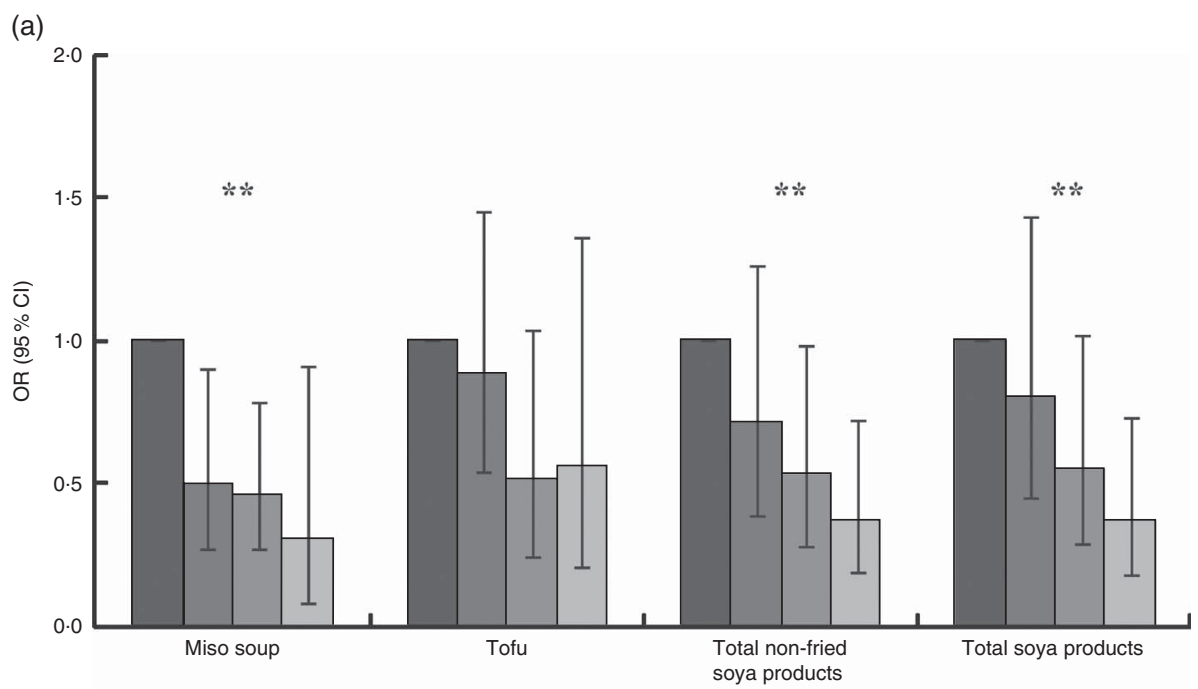

(b)

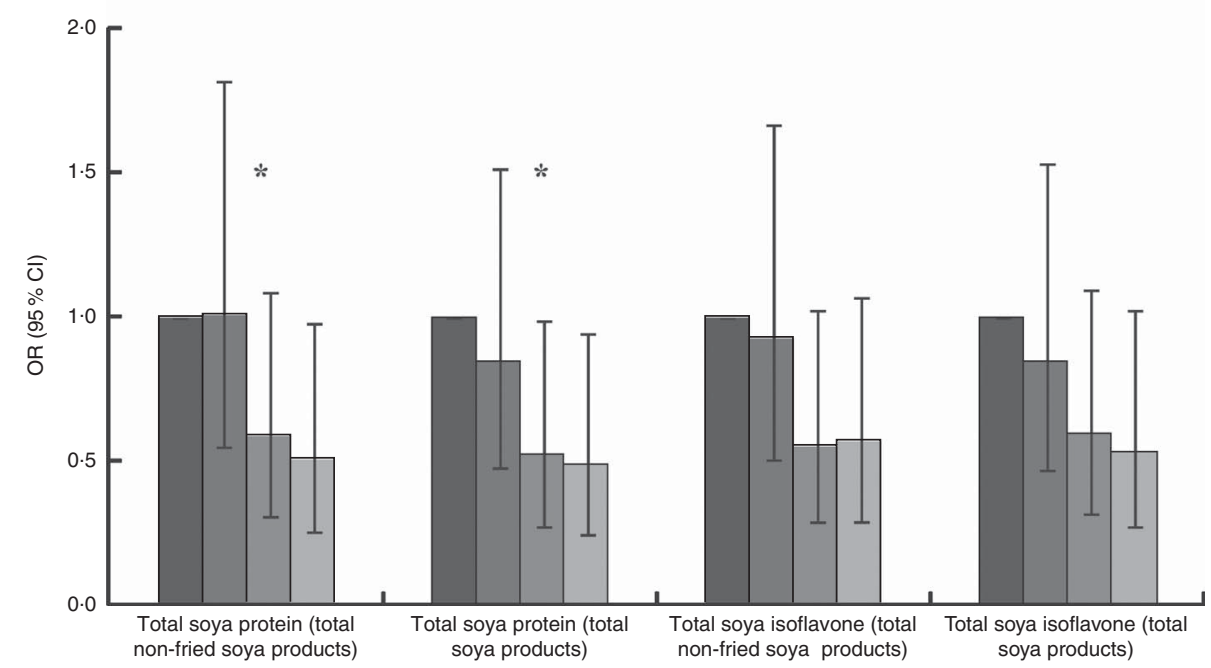

Fig. 2 Association between the intake of each soya product $(1=$ miso soup; $2=$ tofu; $3=$ fermented soybeans and soybeans; $4=$ fried tofu mixed with vegetables, fried bean curd and thick deep-fried tofu) and insulin resistance among 565 men and 583 women aged 34-70 years baseline, living in Tokushima Prefecture between 2008 and 2013, Japan Multi-Institutional Collaborative Cohort (J-MICC) Study. Consumption was divided into quartiles ( $\square$, Q1; $\square$, Q2; $\square$, Q3; $\square$, Q4), with Q1 being defined as the reference. Results are presented as odds ratios with their $95 \%$ profile likelihood confidence intervals represented by vertical bars, and are adjusted for gender, age, recruitment, family history of type 2 diabetes mellitus, total energy intake, physical activity, smoking and drinking habits, and BMI. (a) No significant linear trend was observed for the association between the frequency of intake of tofu and log (OR) of insulin resistance. However, the relationships of intake of miso soup, total non-fried soya products $(1+2+3)$ and total soya products $(1+2+3+4)$ with insulin resistance were significant. (b) The OR of insulin resistance decreased significantly as the estimated intake of soya protein from total non-fried soya products $(1+2+3)$ and total soya products $(1+2+3+4)$ increased $(P$ for trend $<0.05)$. However, the associations between the estimated intake of soya isoflavone from total non-fried soya products $(1+2+3)$ and total soya products $(1+2+3+4)$ and insulin resistance were slightly attenuated. ${ }^{*} P$ for trend $<0.05,{ }^{\star \star} P$ for trend $<0.01$

suggest that the inverse association between soya product intake and insulin resistance was not strongly confounded or intermediated by obesity.

Our study has some limitations. First, the temporal relationship between soya product intake and insulin resistance remains obscure because our study was a crosssectional design. Second, the sample size of the study was small. Third, information on soya product consumption was self-reported and our FFQ did not include information on portion size. However, we used information on soya product intake per meal, which was calculated from the four $3 \mathrm{~d}$ diet records of twenty-eight participants, in order to estimate the intake of soya protein and isoflavone. In consequence, Spearman's rank-correlation coefficient of the estimated intake of soya protein from total non-fried soya products $(1+2+3)$ and total soya products $(1+2+3+4)$, and the estimated intake of soya isoflavone from total non-fried soya products $(1+2+3)$ and total soya 
Table 3 Associations of the estimated intake of soya protein and soya isoflavone with insulin resistance* among 565 men and 583 women aged 34-70 years baseline, living in Tokushima Prefecture between 2008 and 2013, Japan Multi-Institutional Collaborative Cohort (J-MICC) Study

\begin{tabular}{|c|c|c|c|c|c|c|c|c|}
\hline & \multicolumn{7}{|c|}{ Intake amount (g/week) } & \multirow[b]{3}{*}{$P$ for trend } \\
\hline & \multirow{2}{*}{$\frac{\text { Q1 }}{\text { OR }}$} & \multicolumn{2}{|r|}{ Q2 } & \multicolumn{2}{|c|}{ Q3 } & \multicolumn{2}{|c|}{ Q4 } & \\
\hline & & OR & $95 \% \mathrm{Cl}$ & OR & $95 \% \mathrm{Cl}$ & OR & $95 \% \mathrm{Cl}$ & \\
\hline \multicolumn{9}{|c|}{ Total unsweetened and non-fried soya products calculated on soya protein ( $\mathrm{g} /$ week $) \dagger$} \\
\hline No. of cases/participants & $46 / 303$ & & $4 / 253$ & & /306 & & $3 / 286$ & \\
\hline Age- and gender-adjusted modelł & 1.00 & 0.84 & $0.51,1.36$ & 0.50 & $0.30,0.84$ & 0.52 & $0.29,0.88$ & 0.011 \\
\hline Model $1 \S$ & 1.00 & 0.78 & $0.47,1.30$ & 0.48 & $0.28,0.82$ & 0.50 & $0.28,0.88$ & 0.013 \\
\hline Model 2\| & 1.00 & 1.00 & $0.55,1.82$ & 0.59 & $0.31,1.09$ & 0.51 & $0.26,0.97$ & 0.022 \\
\hline \multicolumn{9}{|c|}{ Total soya products calculated on soya protein (g/week) આ } \\
\hline No. of cases/participants & $45 / 287$ & & 7/286 & &.$/ 286$ & & 4/289 & \\
\hline Age- and gender-adjusted modelł & 1.00 & 0.77 & $0.47,1.24$ & 0.46 & $0.26,0.77$ & 0.53 & $0.30,0.91$ & 0.016 \\
\hline Model $1 \S$ & 1.00 & 0.74 & $0.45,1.21$ & 0.45 & $0.26,0.79$ & 0.51 & $0.28,0.90$ & 0.019 \\
\hline Model 2\| & 1.00 & 0.85 & $0.47,1.51$ & 0.52 & $0.27,0.98$ & 0.49 & $0.25,0.95$ & 0.023 \\
\hline \multicolumn{9}{|c|}{ Total unsweetened and non-fried soya products calculated on soya isoflavone (mg/week) ${ }^{* *}$} \\
\hline No. of cases/participants & $47 / 308$ & & $4 / 254$ & & $/ 296$ & & $5 / 290$ & \\
\hline Age- and gender-adjusted modelł & 1.00 & 0.80 & $0.49,1.31$ & 0.45 & $0.26,0.77$ & 0.56 & $0.32,0.94$ & 0.027 \\
\hline Model $1 \S$ & 1.00 & 0.75 & $0.45,1.25$ & 0.44 & $0.25,0.76$ & 0.54 & $0.31,0.94$ & 0.037 \\
\hline Model 2\|l & 1.00 & 0.92 & $0.51,1.66$ & 0.55 & $0.29,1.02$ & 0.56 & $0.29,1.07$ & 0.061 \\
\hline \multicolumn{9}{|c|}{ Total soya products calculated on soya isoflavone $(\mathrm{mg} /$ week $) \dagger \dagger$} \\
\hline No. of cases/participants & $44 / 288$ & & $5 / 268$ & & 3/306 & & 3/286 & \\
\hline Age- and gender-adjusted modelł & 1.00 & 0.81 & $0.49,1.32$ & 0.54 & $0.32,0.90$ & 0.53 & $0.30,0.92$ & 0.020 \\
\hline Model $1 \S$ & 1.00 & 0.76 & $0.45,1.26$ & 0.54 & $0.31,0.91$ & 0.51 & $0.28,0.91$ & 0.024 \\
\hline Model 2II & 1.00 & 0.85 & $0.47,1.54$ & 0.60 & $0 \cdot 32,1 \cdot 10$ & 0.53 & $0.27,1.03$ & 0.050 \\
\hline
\end{tabular}

${ }^{\star}$ Adjusted odds ratios and $95 \%$ profile likelihood confidence intervals.

†Total unsweetened and non-fried soya products calculated on soya protein (g/week): Q1, 0-9.73448; Q2, 9.73448-15.39853; Q3, 15.39853-25.38757; Q4, >25.38757.

$\ddagger$ Age- and gender-adjusted model: adjusted for age (continuous), gender and recruitment (binary).

§Model 1: adjusted for age (continuous), gender, recruitment (binary), family history of type 2 diabetes mellitus (categorical), total energy intake (categorical), physical activity (categorical), smoking (categorical) and drinking habits (binary).

IIModel 2: adjusted for age (continuous), gender, recruitment (binary), family history of type 2 diabetes mellitus (categorical), total energy intake (categorical), physical activity (categorical), smoking (categorical), drinking habits (binary) and BMI (continuous).

TTotal soya products calculated on soya protein (g/week): Q1, 0-11.93971; Q2, 11.93971-18.06136; Q3, 18.06136-28.57594; Q4, >28.57594.

**Total unsweetened and non-fried soya products calculated on soya isoflavone (mg/week): Q1, 0-39.89579; Q2, 39.89579-62.55006; Q3, 62.5500699.25788; Q4, >99.25788.

††Total soya products calculated on soya isoflavone (mg/week): Q1, 0-45.25961; Q2, 45.25961-67.91388; Q3, 67.91388-107.98101; Q4, > 107.98101.

products $(1+2+3+4)$, was $0 \cdot 55(P=0 \cdot 002), 0 \cdot 47(P=0 \cdot 011)$, $0.64(P<0.001)$ and $0.59(P=0.001)$, respectively (see online supplementary material, Supplemental Table 1). On the other hand, the intake of soya products, soya protein or isoflavone may have been underestimated. The median estimated soya isoflavone intake from soya foods based on the National Nutrition Survey of 2002 was $16-22 \mathrm{mg} / \mathrm{d}$, which is equivalent to Q4 in the present study. Fourth, some potential confounders which have a great effect on insulin resistance may have not been completely adjusted for. However, the relationship between insulin resistance and soya products was robust after adjustments for menopause status, vegetable intake or total fibre intake. Finally, because all participants in the study were Japanese, our results may not be applicable to other ethnic groups.

\section{Conclusion}

In conclusion, our results showed that habitual high intake of soyabeans and soya products may be associated with a lower level of insulin resistance. The frequency of the intake of soya products, rather than the estimated intake of soya protein or isoflavone, showed a clearer relationship with insulin resistance. In the future, further studies are needed to clarify the relationship between insulin resistance and metabolites of soya products, using urine and blood samples.

\section{Acknowledgements}

Acknowledgements: The authors thank the following researchers for providing the usable FFQ and a program to calculate nutrient intake: Shinkan Tokudome at the National Institute of Health and Nutrition (formerly Nagoya City University); Chiho Goto at Nagoya Bunri University; Nahomi Imaeda at Nagoya Women's University; Yuko Tokudome at Nagoya University of Arts and Sciences; Masato Ikeda at the University of Occupational and Environmental Health; and Shinzo Maki at Aichi Prefectural Dietetic Association. Financial support: This study was supported in part by Grants-in-Aid for Scientific Research on Priority Areas of Cancer (number 17015018) and on Innovative Areas (number 221S0001) from the Japanese Ministry of Education, Culture, Sports, Science and Technology. The Japanese Ministry of Education, Culture, Sports, Science and Technology had no role in the design, analysis 
or writing of this article. Conflict of interest: None. Authorship: All authors (M.N., H.U., T.S., S.K.-K., M.Y., M.H. and K.A.) developed the idea for the study. M.N., H.U., S.K.-K., M.Y., M.H. and K.A. collected the data. Measurements and data analysis were completed by M.N. and K.A. H.U. and K.A. provided medical advice regarding interpretation of the data. M.N. drafted the manuscript with the help of K.A. All authors approved the final version of the manuscript. Ethics of human subject participation: The study protocol was reviewed and approved by the Ethics Committees of Nagoya University School of Medicine (affiliated with the former principal investigator, Nobuyuki Hamajima), Aichi Cancer Center (affiliated with the current principal investigator, Hideo Tanaka) and the University of Tokushima Graduate School.

\section{Supplementary material}

To view supplementary material for this article, please visit http://dx.doi.org/10.1017/S136898001400247X

\section{References}

1. Wild S, Roglic G, Green A et al. (2004) Global prevalence of diabetes: estimates for the year 2000 and projections for 2030. Diabetes Care 27, 1047-1053.

2. Pinhas-Hamiel O \& Zeitler P (2005) The global spread of type 2 diabetes mellitus in children and adolescents. J Pediatr 146, 693-700.

3. Neville SE, Boye KS, Montgomery WS et al. (2009) Diabetes in Japan: a review of disease burden and approaches to treatment. Diabetes Metab Res Rev 25, 705-716.

4. Molavi B, Rassouli N, Bagwe S et al. (2007) A review of thiazolidinediones and metformin in the treatment of type 2 diabetes with focus on cardiovascular complications. Vasc Health Risk Manag 3, 967-973.

5. Furukawa S, Fujita T, Shimabukuro M et al. (2004) Increased oxidative stress in obesity and its impact on metabolic syndrome. J Clin Invest 114, 1752-1761.

6. Xu H, Barnes GT, Yang Q et al. (2003) Chronic inflammation in fat plays a crucial role in the development of obesityrelated insulin resistance. J Clin Invest 112, 1821-1830.

7. Ren MQ, Kuhn G, Wegner J et al. (2001) Isoflavones, substances with multi-biological and clinical properties. Eur J Nutr 40, 135-146.

8. Vedavanam K, Srijayanta S, O'Reilly J et al. (1999) Antioxidant action and potential antidiabetic properties of an isoflavonoidcontaining soyabean phytochemical extract (SPE). Phytother Res 13, 601-608.

9. Liu D, Zhen W, Yang Z et al. (2006) Genistein acutely stimulates insulin secretion in pancreatic $\beta$-cells through a cAMPdependent protein kinase pathway. Diabetes 55, 1043-1050.

10. Lu MP, Wang R, Song X et al. (2008) Dietary soy isoflavones increase insulin secretion and prevent the development of diabetic cataracts in streptozotocin-induced diabetic rats. Nutr Res 28, 464-471.

11. Ascencio C, Torres N, Isoard-Acosta F et al. (2004) Soy protein affects serum insulin and hepatic SREBP-1 mRNA and reduces fatty liver in rats. J Nutr 134, 522-529.

12. Azadbakht L, Kimiagar M, Mehrabi Y et al. (2007) Soy inclusion in the diet improves features of the metabolic syndrome: a randomized crossover study in postmenopausal women. Am J Clin Nutr 85, 735-741.
13. Li Z, Hong K, Saltsman P et al. (2005) Long-term efficacy of soy-based meal replacements vs an individualized diet plan in obese type II DM patients: relative effects on weight loss, metabolic parameters, and C-reactive protein. Eur J Clin Nutr 59, 411-418.

14. Jayagopal V, Albertazzi P, Kilpatrick ES et al. (2002) Beneficial effects of soy phytoestrogen intake in postmenopausal women with type 2 diabetes. Diabetes Care $\mathbf{2 5}$, 1709-1714.

15. Yang G, Shu XO, Jin F et al. (2004) Soyfood consumption and risk of glycosuria: a cross-sectional study within the Shanghai Women's Health Study. Eur J Clin Nutr 58, 615-620.

16. Nanri A, Mizoue T, Takahashi Y et al. (2010) Soy product and isoflavone intakes are associated with a lower risk of type 2 diabetes in overweight Japanese women. J Nutr $\mathbf{1 4 0}$, 580-586.

17. Villegas R, Gao YT, Yang G et al. (2008) Legume and soy food intake and the incidence of type 2 diabetes in the Shanghai Women's Health Study. Am J Clin Nutr 87, 162-167.

18. Mueller NT, Odegaard AO, Gross MD et al. (2012) Soy intake and risk of type 2 diabetes mellitus in Chinese Singaporeans: soy intake and risk of type 2 diabetes. Eur J Nutr 51, 1033-1040.

19. Morimoto Y, Steinbrecher A, Kolonel LN et al. (2011) Soy consumption is not protective against diabetes in Hawaii: the Multiethnic Cohort. Eur J Clin Nutr 65, 279-282.

20. Hamajima N; J-MICC Study Group (2007) The Japan MultiInstitutional Collaborative Cohort Study (J-MICC Study) to detect gene-environment interactions for cancer. Asian Pac J Cancer Prev 8, 317-323.

21. Hishida A, Morita E, Naito M et al. (2012) Associations of apolipoprotein A5 (APOA5), glucokinase (GCK) and glucokinase regulatory protein (GCKR) polymorphisms and lifestyle factors with the risk of dyslipidemia and dysglycemia in Japanese - a cross-sectional data from the J-MICC Study. Endocr J 59, 589-599.

22. Tokudome S, Goto C, Imaeda N et al. (2004) Development of a data-based short food frequency questionnaire for assessing nutrient intake by middle-aged Japanese. Asian Pac J Cancer Prev 5, 40-43.

23. Tokudome Y, Goto C, Imaeda N et al. (2005) Relative validity of a short food frequency questionnaire for assessing nutrient intake versus three-day weighed diet records in middle-aged Japanese. J Epidemiol 15, 135-145.

24. Goto C, Tokudome Y, Imaeda N et al. (2006) Validation study of fatty acid consumption assessed with a short food frequency questionnaire against plasma concentration in middle-aged Japanese people. Scand J Food Nutr 50, $77-82$.

25. Imaeda N, Goto C, Tokudome Y et al. (2007) Reproducibility of a short food frequency questionnaire for Japanese general population. J Epidemiol 17, 100-107.

26. SAS Institute (1997) SAS/STAT Software: Changes and Enhancements through Release 6.12. Cary, NC: SAS Institute.

27. Liu ZM, Chen YM, Ho SC et al. (2010) Effects of soy protein and isoflavones on glycemic control and insulin sensitivity: a 6-mo double-blind, randomized, placebo-controlled trial in postmenopausal Chinese women with prediabetes or untreated early diabetes. Am J Clin Nutr 91, 1394-1401.

28. Gobert CP, Pipe EA, Capes SE et al. (2010) Soya protein does not affect glycaemic control in adults with type 2 diabetes. Br J Nutr 103, 412-421.

29. Sites CK, Cooper BC, Toth MJ et al. (2007) Effect of a daily supplement of soy protein on body composition and insulin secretion in postmenopausal women. Fertil Steril 88, 1609-1617.

30. Nikander E, Tiitinen A, Laitinen K et al. (2004) Effects of isolated isoflavonoids on lipids, lipoproteins, insulin sensitivity, 
and ghrelin in postmenopausal women. J Clin Endocrinol Metab 89, 3567-3572.

31. Hall WL, Vafeiadou K, Hallund J et al. (2006) Soyisoflavone-enriched foods and markers of lipid and glucose metabolism in postmenopausal women: interactions with genotype and equol production. Am J Clin Nutr 83, 592-600.

32. Bhathena SJ \& Velasquez MT (2002) Beneficial role of dietary phytoestrogens in obesity and diabetes. Am J Clin Nutr 76, 1191-1201.

33. Hamosh M \& Hamosh P (1975) The effect of estrogen on the lipoprotein lipase activity of rat adipose tissue. J Clin Invest 55, 1132-1135.

34. Ørgaard A \& Jensen L (2008) The effects of soy isoflavones on obesity. Exp Biol Med (Maywood) 233, 1066-1080.

35. Naaz A, Yellayi S, Zakroczymski MA et al. (2003) The soy isoflavone genistein decreases adipose deposition in mice. Endocrinology 144, 3315-3320.
36. Wu J, Oka J, Ezaki J et al. (2007) Possible role of equol status in the effects of isoflavone on bone and fat mass in postmenopausal Japanese women: a double-blind, randomized, controlled trial. Menopause 14, 866-874.

37. Arai Y, Uehara M, Sato Y et al. (2000) Comparison of isoflavones among dietary intake, plasma concentration and urinary excretion for accurate estimation of phytoestrogen intake. J Epidemiol 10, 127-135.

38. Lampe JW, Karr SC, Hutchins AM et al. (1998) Urinary equol excretion with a soy challenge: influence of habitual diet. Proc Soc Exp Biol Med 217, 335-339.

39. Liu ZM, Chen YM \& Ho SC (2011) Effects of soy intake on glycemic control: a meta-analysis of randomized controlled trials. Am J Clin Nutr 93, 1092-1101.

40. Ricci E, Cipriani S, Chiaffarino F et al. (2010) Effects of soy isoflavones and genistein on glucose metabolism in perimenopausal and postmenopausal non-Asian women: a meta-analysis of randomized controlled trials. Menopause 17, 1080-1086. 